\title{
An Inequality of Meromorphic Vector Functions and Its Application
}

\author{
Wu Zhaojun ${ }^{1,2}$ and Chen Yuxian ${ }^{2}$ \\ ${ }^{1}$ School of Mathematics and Statistics, Xianning University, Xianning 437100, China \\ ${ }^{2}$ School of Mathematics and Computer Science, Xinyu University, Xinyu 338004, China \\ Correspondence should be addressed to Wu Zhaojun,wuzj52@hotmail.com
}

Received 17 July 2011; Accepted 7 November 2011

Academic Editor: Gerd Teschke

Copyright (c) 2011 W. Zhaojun and C. Yuxian. This is an open access article distributed under the Creative Commons Attribution License, which permits unrestricted use, distribution, and reproduction in any medium, provided the original work is properly cited.

Firstly, an inequality for vector-valued meromorphic functions is established which extend a corresponding inequality of Milloux for meromorphic scalar-valued function (1946). As an application, the relationship between the characteristic function of a vector-valued meromorphic function $f$ and its derivative $f^{\prime}$ is studied, results are obtained to extend some related results for meromorphic scalar-valued function of Weitsman (1969) and Singh and Gopalakrishna (1971).

\section{Introduction of Vector-Valued Meromorphic Function}

In 1980s, Ziegler [1] established Nevanlinna's theory for the vector-valued meromorphic function in finite dimensional spaces. After Ziegler some works related to vector-valued meromorphic function were done in 1990s [2-4]. In this section, we shall introduce the following fundamental notations and results of vector-valued Nevanlinna theory which were quoted from Ziegler [1].

We denote by $\mathbb{C}^{n}$ the usual $n$ dimensional complex Euclidean space with the coordinates $w=\left(w_{1}, w_{2}, \ldots, w_{n}\right)$, the Hermitian scalar product

$$
\langle v, w\rangle=v_{1} \bar{w}_{1}+v_{2} \bar{w}_{2}+\cdots+v_{n} \bar{w}_{n}, \quad\left(v, w \in \mathbb{C}^{n}\right),
$$

and the distance

$$
\|v-w\|=+\langle v-w, v-w\rangle^{1 / 2} .
$$


Let

$$
w_{1}=f_{1}(z), \quad w_{2}=f_{2}(z), \ldots, w_{n}=f_{n}(z)
$$

be $n \geq 1$ complex valued functions of the complex variable $z$, which are meromorphic and not all constant in the Gaussian plane $\mathbb{C}^{1}=\mathbb{C}$, or in a finite disc

$$
\mathbb{C}_{R}=\{|z|<R\} \subset \mathbb{C}, \quad 0<R<+\infty
$$

Thus in $\mathbb{C}_{R}, 0<R \leq+\infty$ (we put $\mathbb{C}_{+\infty}=\mathbb{C}$ ), a vector-valued meromorphic function

$$
f(z)=\left(f_{1}(z), f_{2}(z), \ldots, f_{n}(z)\right)
$$

is given, which does not reduce to the constant zero vector $0=(0,0, \ldots, 0)$. The $j$ th derivative $j=1,2, \ldots$ of $f(z)$ are defined by

$$
f^{(j)}(z)=\left(f_{1}^{(j)}(z), f_{2}^{(j)}(z), \ldots, f_{n}^{(j)}(z)\right)
$$

For such a function, the notations "zero," "pole," and "multiplicity" are defined as in the scalar case $n=1$ of only one meromorphic function $f_{1}(z)$. More explicitly, in the punctured vicinity of each point $z_{0} \in \mathbb{C}_{R}$, the vector function $w=f(z)$ can developed into a Laurent series

$$
f(z)=c_{k_{0}}\left(z-z_{0}\right)^{k_{0}}+c_{k_{0}+1}\left(z-z_{0}\right)^{k_{0}+1}+\cdots,
$$

where the coefficients are vectors

$$
c_{k}=\left(c_{k}^{1}, c_{k}^{2}, \ldots, c_{k}^{n}\right) \in \mathbb{C}^{n}, \quad c_{k_{0}} \neq(0,0, \ldots, 0)
$$

In order to introduce the Nevanlinna theory of vector-valued meromorphic function, we will denote by " $\infty$ " the ideal element of the Aleksandrov one-point compactification of $\mathbb{C}^{n}$ (the two real infinities will be denoted by $+\infty$ and $-\infty$, resp.). Now, if $k_{0} \leq 0$ in the above Laurent expansion, then $z_{0}$ will be called a pole or an $\infty$-point of $f(z)$ of multiplicity $-k_{0}$; in such a point $z_{0}$ at least one of the meromorphic component functions $f_{j}(z)$ has a pole of this multiplicity in the ordinary sense of function theory, so that in $z_{0}$ itself $f(z)$ is not defined. If $k_{0}>0$ in Laurent expansion, then $z_{0}$ is called a zero of $f(z)$ of multiplicity $k_{0}$; in such a point $z_{0}$, all component functions $f_{j}(z)$ vanish, each with at least this multiplicity. 
Let $n(r, f)$ or $n(r, \infty)$ denote the number of poles of $f(z)$ in $|z| \leq r$ and $n(r, a)$ denote the number of $a$-points of $f(z)$ in $|z| \leq r$, counting with multiplicities. Define the volume function associated with vector-valued meromorphic function $f(z)$,

$$
\begin{aligned}
& V(r, \infty)=V(r, f)=\frac{1}{2 \pi} \int_{C_{r}} \log \left|\frac{r}{\xi}\right| \Delta \log \|f(\xi)\| d x \wedge d y, \quad \xi=x+i y \\
& V(r, a)=V\left(r, \frac{1}{f-a}\right)=\frac{1}{2 \pi} \int_{C_{r}} \log \left|\frac{r}{\xi}\right| \Delta \log \|f(\xi)-a\| d x \wedge d y, \quad \xi=x+i y
\end{aligned}
$$

and the counting function of finite or infinite $a$-points by

$$
\begin{aligned}
& N(r, f)=n(0, f) \log r+\int_{0}^{r} \frac{n(t, f)-n(0, f)}{t} d t \\
& N(r, \infty)=n(0, \infty) \log r+\int_{0}^{r} \frac{n(t, \infty)-n(0, \infty)}{t} d t \\
& N(r, a)=n(0, a) \log r+\int_{0}^{r} \frac{n(t, a)-n(0, a)}{t} d t
\end{aligned}
$$

respectively. Next, we define

$$
\begin{gathered}
m(r, \infty)=m(r, f)=\frac{1}{2 \pi} \int_{0}^{2 \pi} \log ^{+}\left\|f\left(r e^{i \theta}\right)\right\| d \theta, \\
m(r, a)=\frac{1}{2 \pi} \int_{0}^{2 \pi} \log ^{+} \frac{1}{\left\|f\left(r e^{i \theta}\right)-a\right\|} d \theta, \\
T(r, f)=m(r, f)+N(r, f) .
\end{gathered}
$$

Let $\bar{n}(r, f)$ or $\bar{n}(r, \infty)$ denote the number of poles of $f(z)$ in $|z| \leq r$ and $\bar{n}(r, a)$ denote the number of $a$-points of $f(z)$ in $|z| \leq r$, ignoring multiplicities. Define the counting function of finite or infinite $a$-points by

$$
\begin{aligned}
& \bar{N}(r, f)=\bar{n}(0, f) \log r+\int_{0}^{r} \frac{\bar{n}(t, f)-\bar{n}(0, f)}{t} d t \\
& \bar{N}(r, \infty)=\bar{n}(0, \infty) \log r+\int_{0}^{r} \frac{\bar{n}(t, \infty)-\bar{n}(0, \infty)}{t} d t \\
& \bar{N}(r, a)=\bar{n}(0, a) \log r+\int_{0}^{r} \frac{\bar{n}(t, a)-\bar{n}(0, a)}{t} d t
\end{aligned}
$$

respectively. 
If $f(z)$ is a vector-valued meromorphic function in the whole complex plane, then the order and the lower order of $f(z)$ are defined by

$$
\begin{aligned}
& \lambda(f)=\limsup _{r \rightarrow \infty} \frac{\log T(r, f)}{\log r}, \\
& \mu(f)=\liminf _{r \rightarrow \infty} \frac{\log T(r, f)}{\log r} .
\end{aligned}
$$

We call the vector-valued meromorphic function $f$ admissible if

$$
\limsup _{r \rightarrow+\infty} \frac{T(r, f)}{\log r}=+\infty \text {. }
$$

Definition 1.1. For a meromorphic function $f(z)$ (vector-valued or scalar-valued), we denote by $S(r, f)$ any quantity such that

$$
S(r, f)=o(T(r, f)), \quad r \longrightarrow+\infty
$$

without restriction if $f(z)$ is of finite order and otherwise except possibly for a set of values of $r$ of finite linear measure.

Definition 1.1 quoted from [2]. In [1], Ziegler established the following first main theorem, logarithmic derivative lemma, and deficient values theorem for meromorphic vector function.

Theorem A. Let $f(z)=\left(f_{1}(z), f_{2}(z), \ldots, f_{n}(z)\right)$ be a meromorphic vector function in $\mathbb{C}_{R}$. Then for $0<r<R \leq+\infty, a \in \mathbb{C}^{n}, f(z) \not \equiv a$, then

$$
T(r, f)=V(r, a)+N(r, a)+m(r, a)+O(1)
$$

Theorem B. Let $f(z)=\left(f_{1}(z), f_{2}(z), \ldots, f_{n}(z)\right)$ be a nonconstant meromorphic vector function in C. Then

$$
\frac{1}{2 \pi} \int_{0}^{2 \pi} \log ^{+} \frac{\left\|f^{\prime}\left(r e^{i \theta}\right)\right\|}{\left\|f\left(r e^{i \theta}\right)-a\right\|} d \theta=S(r, f), \quad a \in \mathbb{C}^{n} .
$$

By the second main theorem, Ziegler [1] studies the following deficiency theorem for meromorphic vector function. For any vector $a \in \mathbb{C}^{n}$, we define the number $\delta(a)=\delta(a, f)$ by putting

$$
\begin{gathered}
\delta(a)=\delta(a, f)=\liminf _{r \rightarrow+\infty} \frac{m(r, a)}{T(r, f)}=1-\limsup _{r \rightarrow+\infty} \frac{V(r, a)+N(r, a)}{T(r, f)}, \\
\delta(\infty)=\delta(\infty, f)=\liminf _{r \rightarrow+\infty} \frac{m(r, f)}{T(r, f)}=1-\limsup _{r \rightarrow+\infty} \frac{N(r, f)}{T(r, f)},
\end{gathered}
$$


and $\Theta(a)=\Theta(a, f)$ by putting

$$
\begin{gathered}
\Theta(a)=\Theta(a, f)=1-\limsup _{r \rightarrow+\infty} \frac{V(r, a)+\bar{N}(r, a)}{T(r, f)}, \\
\Theta(\infty)=\Theta(\infty, f)=1-\limsup _{r \rightarrow+\infty} \frac{\bar{N}(r, f)}{T(r, f)},
\end{gathered}
$$

Theorem C. Let $f(z)=\left(f_{1}(z), f_{2}(z), \ldots, f_{n}(z)\right)$ be an admissible meromorphic vector function in $\mathbb{C}$. Then the set $\left\{a \in \mathbb{C}^{n} \cup\{\infty\}, \delta(a)>0\right\}$ is at most countable and summing over all such points we have

$$
\sum_{a} \delta(a) \leq \sum_{a} \Theta(a) \leq 2
$$

\section{A Fundamental Inequality of Meromorphic Vector Function}

For meromorphic scalar-valued function $f(z)$, Milloux [5] has proved the following theorem.

Theorem D. If $f(z)$ is a nonconstant meromorphic scalar-valued function in Gaussian complex plane $\mathbb{C}$ and if $a_{i}, i=1,2, \ldots, q$, are distinct elements of $\mathbb{C}$ (where $q$ is any positive integer), then

$$
q T(r, f) \leq T\left(r, f^{\prime}\right)+\sum_{i=1}^{q} \bar{N}\left(r, a_{i}\right)+S(r, f) .
$$

For some alternative proofs of Theorem D, see [6] or [7]. It is natural to consider whether there exists a similar results if meromorphic scalar-valued function $f(z)$ is replaced by meromorphic vector-valued function $f(z)$. In this section, the main contribution is to extend Theorem $\mathrm{D}$ to vector-valued meromorphic function by referring the method of $[1,7]$.

Theorem 2.1. Let $f(z)=\left(f_{1}(z), f_{2}(z), \ldots, f_{n}(z)\right)$ be an admissible meromorphic vector function in $\mathbb{C}$ and if $a^{[j]}, j=1,2, \ldots, q$, are distinct elements of $\mathbb{C}^{n}$ (where $q$ is any positive integer), then

$$
q T(r, f) \leq T\left(r, f^{\prime}\right)+\sum_{j=1}^{q}\left(\bar{N}\left(r, a^{[j]}\right)+V\left(r, a^{[j]}\right)\right)+S(r, f) .
$$

Proof. Put

$$
F(z)=\sum_{j=1}^{q} \frac{1}{\left\|f(z)-a^{[j]}\right\|}
$$

We can get

$$
\frac{1}{2 \pi} \int_{0}^{2 \pi} \log ^{+} F\left(r e^{i \theta}\right) d \theta \leq m\left(r, 0, f^{\prime}\right)+\frac{1}{2 \pi} \int_{0}^{2 \pi} \log ^{+}\left\{F\left(r e^{i \theta}\right)\left\|f^{\prime}\left(r e^{i \theta}\right)\right\|\right\} d \theta .
$$


Put

$$
\delta=\min _{i \neq j}\left\|a^{[i]}-a^{[j]}\right\|
$$

Let for the moment $\mu \in\{1,2, \ldots, q\}$ be fixed. Then we get in every point where

$$
\left\|f(z)-a^{[\mu]}\right\|<\frac{\delta}{2 q} \leq \frac{\delta}{4}
$$

the inequality

$$
\left\|f(z)-a^{[v]}\right\| \geq\left\|a^{[\mu]}-a^{[v]}\right\|-\left\|f(z)-a^{[\mu]}\right\| \geq \frac{3 \delta}{4}
$$

for $\mu \neq v$. Therefore, the set of points on $\partial \mathbb{C}_{r}$ which is determined by (2.6) is either empty or any two such sets for different $\mu$ have empty intersection. In any case,

$$
\begin{gathered}
\frac{1}{2 \pi} \int_{0}^{2 \pi} \log ^{+} F\left(r e^{i \theta}\right) d \theta \geq \frac{1}{2 \pi} \sum_{\mu=1}^{q} \int_{\left\|f(z)-a^{[\mu]}\right\|<\delta / 2 q,|z|=r} \log ^{+} F\left(r e^{i \theta}\right) d \theta \\
\quad \geq \frac{1}{2 \pi} \sum_{\mu=1}^{q} \int_{\left\|f(z)-a^{[\mu]}\right\|<\delta / 2 q,|z|=r} \log ^{+} \frac{1}{\left\|f\left(r e^{i \theta}\right)-a^{[\mu]}\right\|} d \theta .
\end{gathered}
$$

Because of

$$
\begin{aligned}
& \frac{1}{2 \pi} \int_{\left\|f(z)-a^{[\mu]}\right\|<\delta / 2 q,|z|=r} \log ^{+} \frac{1}{\left\|f\left(r e^{i \theta}\right)-a^{[\mu]}\right\|} d \theta \\
& \quad=m\left(r, a^{[\mu]}\right)-\frac{1}{2 \pi} \int_{\left\|f(z)-a^{[\mu]}\right\| \geq \delta / 2 q,|z|=r} \log ^{+} \frac{1}{\left\|f\left(r e^{i \theta}\right)-a^{[\mu]}\right\|} d \theta \\
& \quad \geq m\left(r, a^{[\mu]}\right)-\log ^{+} \frac{2 q}{\delta},
\end{aligned}
$$

it follows that

$$
\frac{1}{2 \pi} \int_{0}^{2 \pi} \log ^{+} F\left(r e^{i \theta}\right) d \theta \geq \sum_{\mu=1}^{q} m\left(r, a^{[\mu]}\right)-\log ^{+} \frac{2 q}{\delta}
$$

so that by (2.4)

$$
\sum_{\mu=1}^{q} m\left(r, a^{[\mu]}\right) \leq m\left(r, 0, f^{\prime}\right)+\frac{1}{2 \pi} \int_{0}^{2 \pi} \log ^{+}\left\{F\left(r e^{i \theta}\right)\left\|f^{\prime}\left(r e^{i \theta}\right)\right\|\right\} d \theta+\log ^{+} \frac{2 q}{\delta}
$$


Abstract and Applied Analysis

Thus by Theorem B, we have

$$
\sum_{\mu=1}^{q} m\left(r, a^{[\mu]}\right) \leq m\left(r, 0, f^{\prime}\right)+S(r, f)
$$

It follows from Theorem A that

$$
m\left(r, 0, f^{\prime}\right)+N\left(r, 0, f^{\prime}\right)+V\left(r, 0, f^{\prime}\right)=T\left(r, f^{\prime}\right)+O(1)
$$

Thus from (2.12) and (2.13), we deduce

$$
\sum_{\mu=1}^{q} m\left(r, a^{[\mu]}\right) \leq T\left(r, f^{\prime}\right)-N\left(r, 0, f^{\prime}\right)+S(r, f) .
$$

Adding $\sum_{\mu=1}^{q} N\left(r, a^{[\mu]}\right)$ to both sides,

$$
\begin{aligned}
\sum_{\mu=1}^{q} T\left(r, \frac{1}{f-a^{[\mu]}}\right) & \leq T\left(r, f^{\prime}\right)+\sum_{\mu=1}^{q} N\left(r, a^{[\mu]}\right)-N\left(r, 0, f^{\prime}\right)+S(r, f) \\
& =T\left(r, f^{\prime}\right)+\sum_{\mu=1}^{q} \bar{N}\left(r, a^{[\mu]}\right)-N_{0}\left(r, 0, f^{\prime}\right)+S(r, f)
\end{aligned}
$$

where $N_{0}\left(r, 0, f^{\prime}\right)$ is formed with the zeros of $f^{\prime}$ which are not zeros of any of $f-a^{[\mu]},(i=$ $1,2, \ldots, q)$. Since $N_{0}\left(r, 0, f^{\prime}\right) \geq 0$, we have

$$
\sum_{\mu=1}^{q} T\left(r, \frac{1}{f-a^{[\mu]}}\right) \leq T\left(r, f^{\prime}\right)+\sum_{\mu=1}^{q} \bar{N}\left(r, a^{[\mu]}\right)+S(r, f) .
$$

Since

$$
T\left(r, \frac{1}{f-a^{[\mu]}}\right)+V\left(r, a^{[\mu]}\right)=T(r, f)+O(1)
$$

it follows that

$$
q T(r, f) \leq T\left(r, f^{\prime}\right)+\sum_{j=1}^{q}\left(\bar{N}\left(r, a^{[j]}\right)+V\left(r, a^{[j]}\right)\right)+S(r, f)
$$




\section{Characteristic Function of Derivative of Meromorphic Vector Function}

Let $f(z)$ be a meromorphic scalar-valued function in $\mathbb{C}$. The characteristic function of derivative of $f(z)$ with $\sum_{a} \delta(a)=2$ has been studied by Edrei [8], Shan and Singh [9], Singh and Gopalakrishna [7], Singh and Kulkarni [10] and Weitsman [11]. For example, Edrei [8] and Weitsman [11] have proved the following theorem.

Theorem E. Let $f(z)$ be a transcendental meromorphic scalar-valued function of finite order and assume $\sum_{a \in \mathbb{C}} \delta(a)=\eta \geq 1$ and $\delta(\infty)=2-\eta$. Then

$$
T\left(r, f^{\prime}\right) \sim \eta T(r, f), \quad r \longrightarrow+\infty
$$

If $\sum_{a} \delta(a)=2$ is replaced by $\sum_{a} \Theta(a)=2$, Singh and Gopalakrishna [7] and Singh and Kulkarni [10] have proved the following theorem.

Theorem F. Let $f(z)$ be a transcendental meromorphic scalar-valued function of finite order and assume $\sum_{a} \Theta(a)=2$. Then

$$
\begin{aligned}
& \lim _{r \rightarrow+\infty} \frac{T\left(r, f^{\prime}\right)}{T(r, f)}=2-\Theta(\infty), \\
& \lim _{r \rightarrow+\infty} \frac{\bar{N}(r, a)}{T(r, f)}=1-\Theta(a)
\end{aligned}
$$

for every $a \in \mathbb{C} \cup\{\infty\}$.

It is natural to consider whether there exists a similar results if meromorphic scalarvalued function $f(z)$ is replaced by meromorphic vector-valued function $f(z)$. In this section, the main purpose is to extend the above theorems to vector-valued meromorphic function by referring the method of $[1,7]$.

Theorem 3.1. Let $f(z)=\left(f_{1}(z), f_{2}(z), \ldots, f_{n}(z)\right)$ be an admissible meromorphic vector function of finite order in $\mathbb{C}$ and assume $\sum_{a} \Theta(a)=2$. Then

$$
\begin{gathered}
\lim _{r \rightarrow+\infty} \frac{T\left(r, f^{\prime}\right)}{T(r, f)}=2-\Theta(\infty), \\
\lim _{r \rightarrow+\infty} \frac{\bar{N}(r, f)}{T(r, f)}=1-\Theta(\infty), \quad \lim _{r \rightarrow+\infty} \frac{\bar{N}(r, a)+V(r, a)}{T(r, f)}=1-\Theta(a)
\end{gathered}
$$

for every $a \in \mathbb{C}^{n}$. 
Proof. Now, basic estimates in vector-valued Nevanlinna theory [1] or [4] yields

$$
\begin{aligned}
T\left(r, f^{\prime}\right) & =m\left(r, f^{\prime}\right)+N\left(r, f^{\prime}\right) \\
& =m\left(r, \frac{f f^{\prime}}{f}\right)+N\left(r, f^{\prime}\right) \\
& \leq m\left(r, \frac{f^{\prime}}{f}\right)+m(r, f)+N(r, f)+\bar{N}(r, f) \\
& \leq T(r, f)+\bar{N}(r, f)+m\left(r, \frac{f^{\prime}}{f}\right) .
\end{aligned}
$$

By Theorem B and the above inequality, we have

$$
\limsup _{r \rightarrow+\infty} \frac{T\left(r, f^{\prime}\right)}{T(r, f)} \leq 2-\Theta(\infty)
$$

Let $\left\{a^{[j]}\right\}$ be a sequence of distinct vector in $\mathbb{C}^{n}$ containing all the vector of $\delta\left(a^{[j]}\right)>0$. From Theorem 2.1, for any positive integer $q$, we have

$$
q T(r, f) \leq T\left(r, f^{\prime}\right)+\sum_{j=1}^{q}\left(\bar{N}\left(r, a^{[j]}\right)+V\left(r, a^{[j]}\right)\right)+S(r, f)
$$

Hence

$$
\begin{aligned}
q & \leq \liminf _{r \rightarrow+\infty} \frac{T\left(r, f^{\prime}\right)}{T(r, f)}+\sum_{j=1}^{q} \limsup _{r \rightarrow+\infty} \frac{\bar{N}\left(r, a^{[j]}\right)+V\left(r, a^{[j]}\right)}{T(r, f)}+\limsup _{r \rightarrow+\infty} \frac{S(r, f)}{T(r, f)} \\
& =\liminf _{r \rightarrow+\infty} \frac{T\left(r, f^{\prime}\right)}{T(r, f)}+\sum_{j=1}^{q}\left\{1-\Theta\left(a^{[j]}\right)\right\}+\limsup _{r \rightarrow+\infty} \frac{S(r, f)}{T(r, f)} .
\end{aligned}
$$

Thus

$$
\liminf _{r \rightarrow+\infty} \frac{T\left(r, f^{\prime}\right)}{T(r, f)} \geq \sum_{j=1}^{q} \Theta\left(a^{[j]}\right)
$$

Since $q$ was arbitrary, we have

$$
2-\Theta(\infty)=\sum_{a \in \mathbb{C}^{n}} \Theta(a) \leq \liminf _{r \rightarrow+\infty} \frac{T\left(r, f^{\prime}\right)}{T(r, f)}
$$


This and (3.5) yield

$$
\lim _{r \rightarrow+\infty} \frac{T\left(r, f^{\prime}\right)}{T(r, f)}=2-\Theta(\infty)
$$

Let $a \in \mathbb{C}^{n} \cup\{\infty\}$ and $\left\{a^{[i]}\right\}_{i=1}^{+\infty}$ an infinite sequence of distinct elements of $\mathbb{C}^{n} \cup\{\infty\}$ which includes every $b \in \mathbb{C}^{n} \cup\{\infty\}$ satisfying $b \neq a$ and $\Theta(b)>0$. Then

$$
\sum_{i=1}^{+\infty} \Theta\left(a^{[i]}\right)=\sum_{b \in \mathbb{C}^{n} \cup\{\infty\}, b \neq a} \Theta(b)=2-\Theta(a) .
$$

Let $q$ be any integer $\geq 3$. From Generalized Second Main Theorem (see [1], Page 126), we have

$$
(q-2) T(r, f)=\sum_{i=1}^{q-1}\left(\bar{N}\left(r, a^{[i]}\right)+V\left(r, a^{[i]}\right)\right)+\bar{N}(r, f)+S(r, f) .
$$

Hence

$$
q-2 \leq \sum_{i=1}^{q-1}\left\{1-\Theta\left(a^{[i]}\right)\right\}+\liminf _{r \rightarrow+\infty} \frac{\bar{N}(r, f)}{T(r, f)}
$$

Thus

$$
\sum_{i=1}^{q-1} \Theta\left(a^{[i]}\right)-1 \leq \liminf _{r \rightarrow+\infty} \frac{\bar{N}(r, f)}{T(r, f)}
$$

Since this holds for all $q \geq 3$, letting $q \rightarrow+\infty$ and combining (3.11), we get

$$
1-\Theta(\infty)=\sum_{i=1}^{+\infty} \Theta\left(a^{[i]}\right)-1 \leq \liminf _{r \rightarrow+\infty} \frac{\bar{N}(r, f)}{T(r, f)} \leq \limsup _{r \rightarrow+\infty} \frac{\bar{N}(r, f)}{T(r, f)}=1-\Theta(\infty) .
$$

So

$$
\lim _{r \rightarrow+\infty} \frac{\bar{N}(r, f)}{T(r, f)}=1-\Theta(\infty)
$$

For every $a \in \mathbb{C}^{n}$, Let $q$ be any integer $\geq 3$. From Generalized Second Main Theorem (see [1], Page 126), we have

$$
(q-2) T(r, f)=\sum_{i=1}^{q-2}\left(\bar{N}\left(r, a^{[i]}\right)+V\left(r, a^{[i]}\right)\right)+(\bar{N}(r, a)+V(r, a))+\bar{N}(r, f)+S(r, f) .
$$


Hence

$$
q-2 \leq \sum_{i=1}^{q-2}\left\{1-\Theta\left(a^{[i]}\right)\right\}+(1-\Theta(\infty))+\liminf _{r \rightarrow+\infty} \frac{\bar{N}(r, a)+V(r, a)}{T(r, f)}
$$

Thus

$$
\sum_{i=1}^{q-2} \Theta\left(a^{[i]}\right)+\Theta(\infty)-1 \leq \liminf _{r \rightarrow+\infty} \frac{\bar{N}(r, a)+V(r, a)}{T(r, f)}
$$

Since this holds for all $q \geq 3$, letting $q \rightarrow+\infty$ and combining (3.11), we get

$$
\begin{aligned}
1-\Theta(a) & =\sum_{i=1}^{+\infty} \Theta\left(a^{[i]}\right)-1 \leq \liminf _{r \rightarrow+\infty} \frac{\bar{N}(r, a)+V(r, a)}{T(r, f)} \\
& \leq \limsup _{r \rightarrow+\infty} \frac{\bar{N}(r, a)+V(r, a)}{T(r, f)}=1-\Theta(a) .
\end{aligned}
$$

So

$$
\lim _{r \rightarrow+\infty} \frac{\bar{N}(r, a)+V(r, a)}{T(r, f)}=1-\Theta(a)
$$

From Theorem 3.1, we have the following corollary

Corollary 3.2. Let $f(z)=\left(f_{1}(z), f_{2}(z), \ldots, f_{n}(z)\right)$ be an admissible meromorphic vector function of finite order in $\mathbb{C}$ and assume $\sum_{a \in \mathbb{C}^{n}} \Theta(a)=2$. Then

$$
T\left(r, f^{\prime}\right) \sim 2 T(r, f), \quad r \longrightarrow+\infty .
$$

Corollary 3.3. Let $f(z)=\left(f_{1}(z), f_{2}(z), \ldots, f_{n}(z)\right)$ be an admissible meromorphic vector function of finite order in $\mathbb{C}$ and assume $\sum_{a \in \mathbb{C}^{n}} \Theta(a)=\eta \geq 1$ and $\delta(\infty)=2-\eta$. Then

$$
T\left(r, f^{\prime}\right) \sim \eta T(r, f), \quad r \longrightarrow+\infty .
$$

Corollary 3.4. Let $f(z)=\left(f_{1}(z), f_{2}(z), \ldots, f_{n}(z)\right)$ be an admissible meromorphic vector function of finite order in $\mathbb{C}$ and assume $\sum_{a} \delta(a)=2$. Then

$$
\begin{gathered}
\lim _{r \rightarrow+\infty} \frac{T\left(r, f^{\prime}\right)}{T(r, f)}=2-\delta(\infty), \\
\lim _{r \rightarrow+\infty} \frac{N(r, f)}{T(r, f)}=1-\delta(\infty), \quad \lim _{r \rightarrow+\infty} \frac{N(r, a)+V(r, a)}{T(r, f)}=1-\delta(a)
\end{gathered}
$$

for every $a \in \mathbb{C}^{n}$. 
Proof. Since $\delta(a) \leq \Theta(a)$ for every $a \in \mathbb{C}^{n} \cup\{\infty\}$ and Theorem C, it follows that, if $\sum_{a} \delta(a)=2$, then $\sum_{a} \Theta(a)=2$ and $\delta(a)=\Theta(a)$ for every $a \in \mathbb{C}^{n} \cup\{\infty\}$. Hence

$$
\lim _{r \rightarrow+\infty} \frac{T\left(r, f^{\prime}\right)}{T(r, f)}=2-\delta(\infty)
$$

follows by Theorem 3.1.

Now, for every $a \in \mathbb{C}^{n}$,

$$
\lim _{r \rightarrow+\infty} \frac{\bar{N}(r, a)+V(r, a)}{T(r, f)}=1-\Theta(a)=1-\delta(a)
$$

Further

$$
\bar{N}(r, a) \leq N(r, a) .
$$

Hence

$$
\begin{aligned}
1-\delta(a) & \leq \lim _{r \rightarrow+\infty} \frac{\bar{N}(r, a)+V(r, a)}{T(r, f)} \\
& \leq \liminf _{r \rightarrow+\infty} \frac{N(r, a)+V(r, a)}{T(r, f)} \\
& \leq \limsup _{r \rightarrow+\infty} \frac{N(r, a)+V(r, a)}{T(r, f)} \\
& =1-\delta(a) .
\end{aligned}
$$

Similarly,

$$
\lim _{r \rightarrow+\infty} \frac{\bar{N}(r, f)}{T(r, f)}=1-\Theta(\infty)=1-\delta(\infty)
$$

Further

$$
\bar{N}(r, \infty) \leq N(r, \infty)
$$

Hence

$$
1-\delta(\infty) \leq \lim _{r \rightarrow+\infty} \frac{\bar{N}(r, \infty)}{T(r, f)} \leq \liminf _{r \rightarrow+\infty} \frac{N(r, \infty)}{T(r, f)} \leq \limsup _{r \rightarrow+\infty} \frac{N(r, \infty)}{T(r, f)}=1-\delta(\infty)
$$

From Corollary 3.4, we have the following corollary. 
Corollary 3.5. Let $f(z)=\left(f_{1}(z), f_{2}(z), \ldots, f_{n}(z)\right)$ be an admissible meromorphic vector function of finite order in $\mathbb{C}$ and assume $\sum_{a \in \mathbb{C}^{n}} \delta(a)=2$. Then

$$
T\left(r, f^{\prime}\right) \sim 2 T(r, f), \quad r \longrightarrow+\infty
$$

Corollary 3.6. Let $f(z)=\left(f_{1}(z), f_{2}(z), \ldots, f_{n}(z)\right)$ be an admissible meromorphic vector function of finite order in $\mathbb{C}$ and assume $\sum_{a \in \mathbb{C}^{n}} \delta(a)=\eta \geq 1$ and $\delta(\infty)=2-\eta$. Then

$$
T\left(r, f^{\prime}\right) \sim \eta T(r, f), \quad r \longrightarrow+\infty
$$

\section{Acknowledgments}

This research was partially supported by the NSF of Jiangxi Province (Grant 2010GZC0187), by NSF of Educational Department of the Hubei Province (Grant T201009, Q20112807), and by Grant PY1002 of Xianning University.

\section{References}

[1] H. J. W. Ziegler, Vector Valued Nevanlinna Theory, vol. 73 of Research Notes in Mathematics, Pitman, Boston, Mass, USA, 1982.

[2] I. Lahiri, "Milloux theorem and deficiency of vector-valued meromorphic functions," The Journal of the Indian Mathematical Society, vol. 55, no. 1-4, pp. 235-250, 1990.

[3] I. Lahiri, "Generalisation of an inequality of C. T. Chuang to vector meromorphic functions," Bulletin of the Australian Mathematical Society, vol. 46, no. 2, pp. 317-333, 1992.

[4] I. Lahiri, "Milloux theorem, deficiency and fix-points for vector-valued meromorphic functions," The Journal of the Indian Mathematical Society, vol. 59, no. 1-4, pp. 45-60, 1993.

[5] H. Milloux, "Les dérivées des fonctions méromorphes et la théorie des défauts," Annales Scientifiques de l'École Normale Supérieure, vol. 63, pp. 289-316, 1947.

[6] H. Wittich, Neuere Untersuchungen über Eindeutige Analytische Funktionen, Springer, Berlin, Germany, 1955.

[7] S. K. Singh and H. S. Gopalakrishna, "Exceptional values of entire and meromorphic functions," Mathematische Annalen, vol. 191, pp. 121-142, 1971.

[8] A. Edrei, "Sums of deficiencies of meromorphic functions. II," Journal d'Analyse Mathématique, vol. 19, pp. 53-74, 1967.

[9] S. M. Shah and S. K. Singh, "On the derivative of a meromorphic function with maximum defect," Mathematische Zeitschrift, vol. 65, pp. 171-174, 1956.

[10] S. K. Singh and V. N. Kulkarni, "Characteristic function of a meromorphic function and its derivative," Annales Polonici Mathematici, vol. 28, pp. 123-133, 1973.

[11] A. Weitsman, "Meromorphic functions with maximal deficiency sum and a conjecture of F. Nevanlinna," Acta Mathematica, vol. 123, pp. 115-139, 1969. 


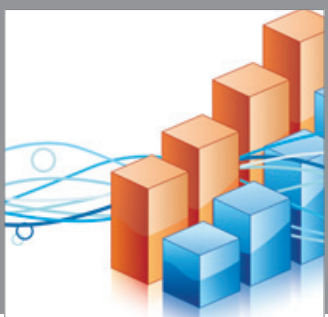

Advances in

Operations Research

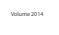

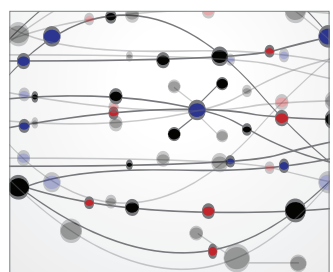

\section{The Scientific} World Journal
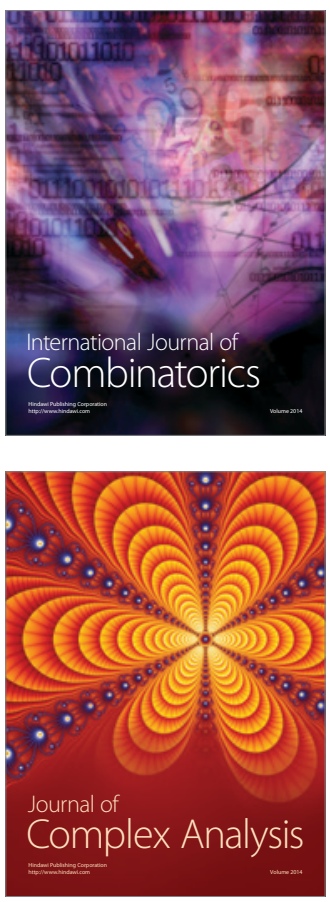

International Journal of

Mathematics and

Mathematical

Sciences
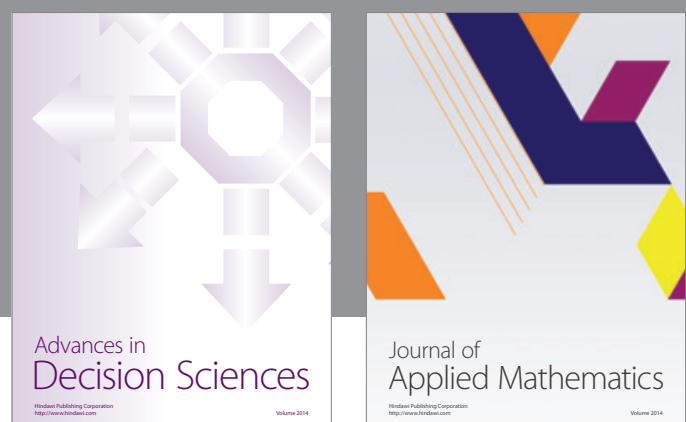

Journal of

Applied Mathematics
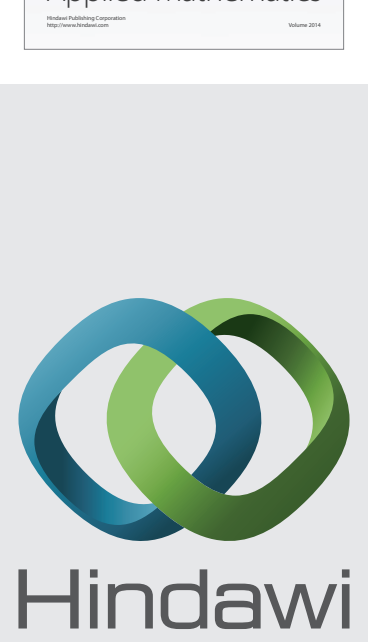

Submit your manuscripts at http://www.hindawi.com
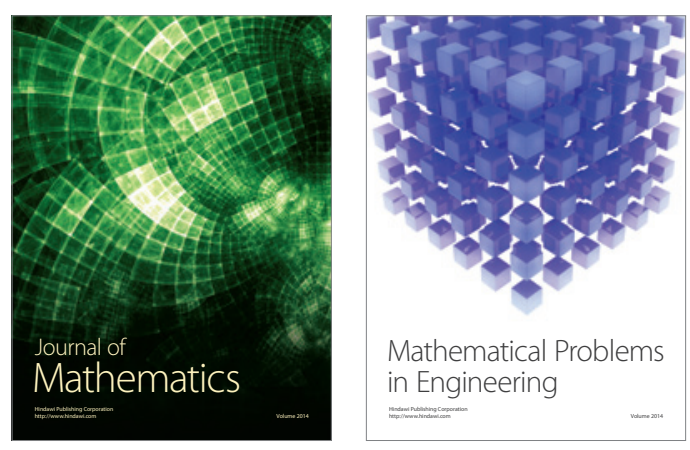

Mathematical Problems in Engineering
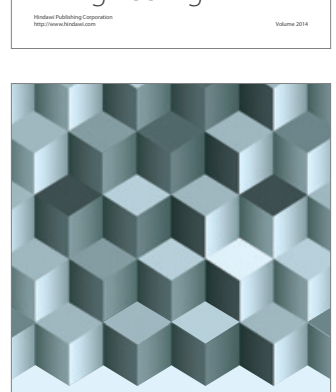

Journal of

Function Spaces
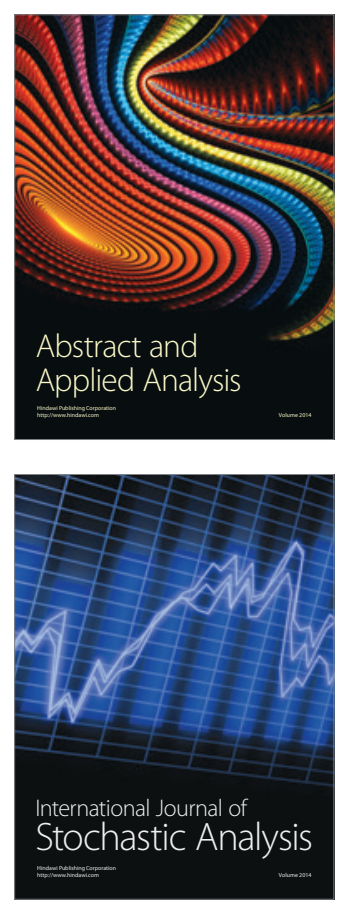

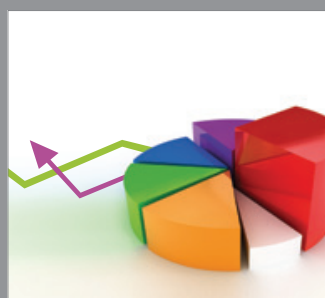

ournal of

Probability and Statistics

Promensencen
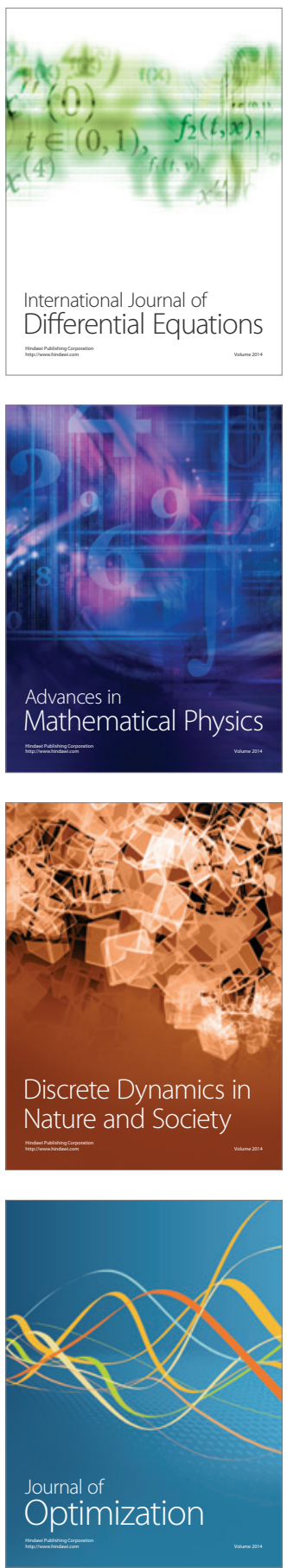